Doug Geisler, Eva K. Grebel, and Dante Minniti, eds.

\title{
Oxygen Abundances in Bulge-like Dwarf Stars
}

\author{
Luciana Pompéia and Beatriz Barbuy \\ Instituto Astronômico e Geofísico, USP, 01060-970 São Paulo, Brazil \\ Michel Grenon \\ Geneva Observatory, Chemin des Maillettes 51, CH-1290 Sauverny, \\ Switzerland
}

\begin{abstract}
High resolution échelle spectra were obtained with the FEROS spectrograph at the $1.5 \mathrm{~m}$ ESO telescope for 35 nearby bulge-like stars. From Hipparcos data it appears that the turn-off of this population indicates an age of 10-11 Gyr (Grenon 2000).

Oxygen abundances are calculated using the [O I] line at $6300.3 \AA$. The derived results show an oxygen overabundance for most of our sample stars when compared to their disk counterparts.
\end{abstract}

\section{Introduction}

Solar neighborhood stars with highly eccentric orbits spanning the metallicity range $-0.5 \leq[\mathrm{Fe} / \mathrm{H}] \leq+0.26$ are studied. These stars were selected according to their kinematics and metallicities as described by Grenon (2000), and they are possible members of the bulge or inner disk.

Oxygen abundances were derived in previous studies for two sub-samples with the given kinematic characteristics (Barbuy \& Grenon 1990; Castro et al. 1997). Barbuy \& Grenon (1990) found a mean value of $[\mathrm{O} / \mathrm{Fe}] \approx+0.2$ dex for 11 stars. Castro et al. (1997) found $[\mathrm{O} / \mathrm{Fe}] \approx 0.0$ dex for a smaller sub-sample.

\section{Observations and Analysis}

Sample stars were observed at the $1.52 \mathrm{~m}$ telescope of ESO, La Silla, with the Fiber Fed Extended Range Optical Spectrograph (FEROS). Using a special package for reductions (DRS) of FEROS data, in MIDAS environment, the data reduction proceeded with subtraction of bias and scattered light in the CCD, orders extraction, flat fielding, and wavelength calibration with a $\mathrm{ThAr}$ calibration frame.

The atmospheric parameters were determined as follows. The effective temperatures were calculated by fitting the $\mathrm{H} \alpha$ wing profiles, which are sensitive to temperature changes. Surface gravities were inferred by requiring ionization equilibrium of $\mathrm{Fe} \mathrm{I}$ and $\mathrm{Fe}$ II lines. Metallicities and microturbulent velocities were derived by using the curves of growth of Fe I and Fe II. In Table 1 the derived parameters are given. 

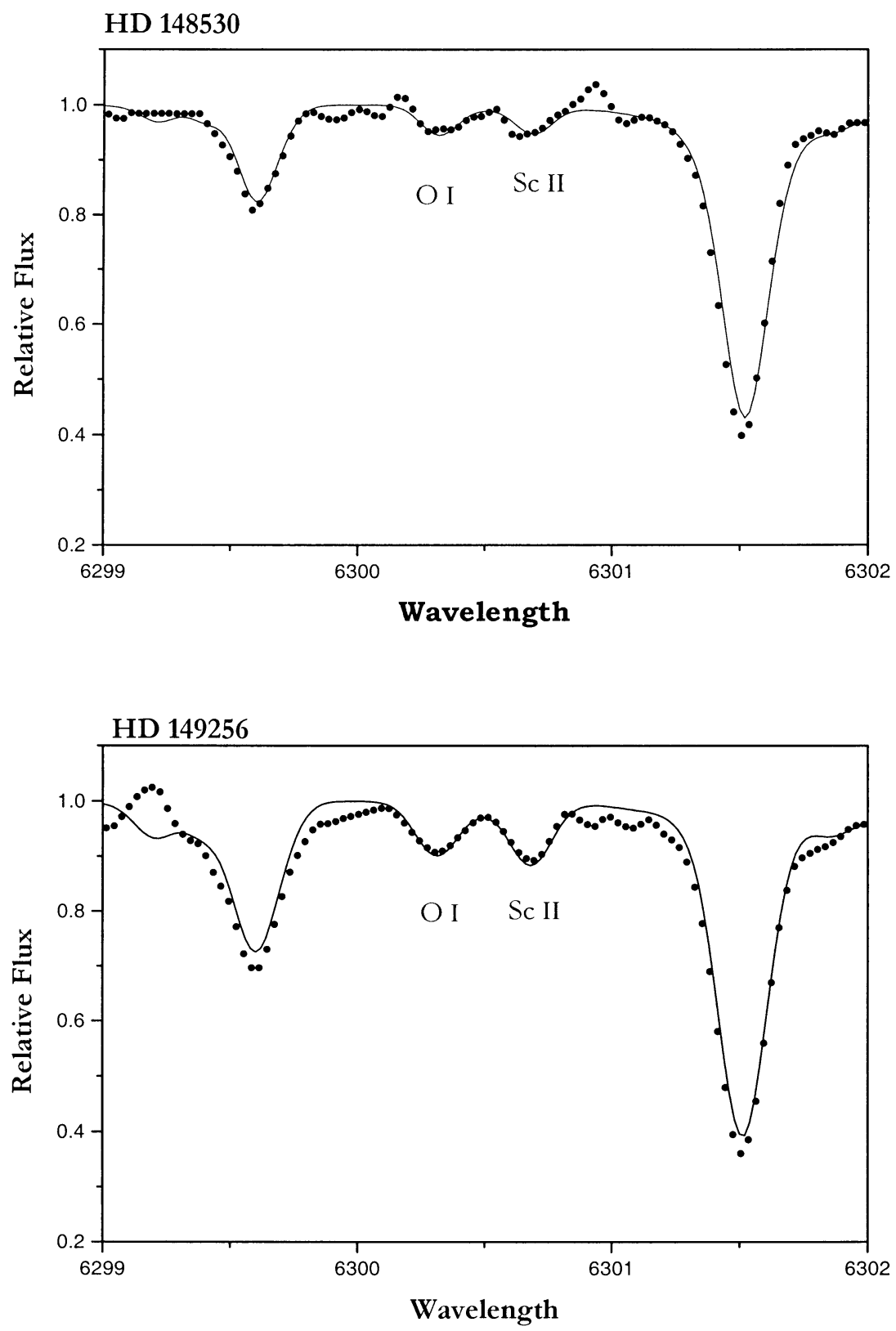

Figure 1. Spectrum synthesis of the [OI] line: observed (circles) and synthetic (solid lines) for HD 148530 and HD 149256. 
Table 1. Atmospheric Parameters

\begin{tabular}{crrrrrrrrr}
\hline Star & $\mathrm{T}_{\text {eff }}$ & $\log g$ & {$[\mathrm{Fe} / \mathrm{H}]$} & $\xi_{t}$ & Star & $\mathrm{T}_{\text {eff }}$ & $\log g$ & {$[\mathrm{Fe} / \mathrm{H}]$} & $\xi_{t}$ \\
\hline HD 143016 & 5575 & 3.8 & -0.50 & 1.0 & CD-4015036 & 5350 & 4.1 & -0.10 & 0.5 \\
HD 143102 & 5500 & 3.7 & 0.10 & 0.9 & HD 219180 & 5400 & 4.4 & -0.70 & 0.5 \\
HD 148530 & 5350 & 4.3 & 0.00 & 0.5 & HD 220536 & 5850 & 3.9 & -0.22 & 1.0 \\
HD 149256 & 5350 & 3.6 & 0.26 & 1.1 & HD 220993 & 5600 & 4.0 & -0.30 & 0.7 \\
HD 152391 & 5300 & 3.9 & -0.12 & 0.9 & HD 224383 & 5800 & 4.1 & -0.02 & 1.0 \\
HDE326583 & 5600 & 3.7 & -0.50 & 0.6 & HD 4308 & 5600 & 4.0 & -0.40 & 0.7 \\
HD 175617 & 5550 & 4.7 & -0.48 & 0.5 & HD 6734 & 5000 & 3.1 & -0.53 & 0.8 \\
HD 178737 & 5575 & 4.0 & -0.33 & 0.6 & HD 8638 & 5500 & 4.1 & -0.50 & 0.9 \\
HD 179764 & 5450 & 4.2 & 0.05 & 0.5 & HD 9424 & 5350 & 4.0 & 0.00 & 0.8 \\
HD 181234 & 5350 & 4.1 & 0.38 & 0.8 & HD 10576 & 5850 & 3.6 & -0.12 & 1.2 \\
HD 184846 & 5600 & 4.0 & -0.25 & 0.8 & HD 10785 & 5850 & 4.2 & -0.25 & 1.0 \\
BD-176035 & 4750 & 3.8 & 0.05 & 1.0 & HD 11306 & 5200 & 4.3 & -0.60 & 0.6 \\
HD 198245 & 5650 & 4.3 & -0.65 & 0.5 & HD 11397 & 5400 & 4.0 & -0.70 & 0.6 \\
HD 201237 & 4950 & 4.3 & -0.05 & 0.5 & HD 14282 & 5800 & 3.7 & -0.40 & 1.0 \\
HD 211276 & 5500 & 4.0 & -0.55 & 0.5 & HD 16623 & 5700 & 4.0 & -0.60 & 1.0 \\
HD 211532 & 5350 & 4.7 & -0.70 & 0.5 & BD-02 603 & 5450 & 4.2 & -0.80 & 0.5 \\
HD 211706 & 5800 & 3.7 & -0.05 & 1.0 & HD 21543 & 5650 & 4.1 & -0.55 & 0.5 \\
HD 214059 & 5550 & 3.8 & -0.33 & 0.6 & & & & & \\
\hline
\end{tabular}

\section{Oxygen abundances and discussion}

Oxygen abundances were derived by comparing the observed [O I] line at 6300.3 $\AA$ to synthetic spectra. The spectrum synthesis code is described in Cayrel et al. (1991). In Figure 1 we show the fit of the [O I] line for HD 149256 and HD 148530. In Table 2 the resulting oxygen abundances are given.

In Figure 2 the $[\mathrm{O} / \mathrm{Fe}]$ vs. $[\mathrm{Fe} / \mathrm{H}]$ relation is plotted for bulge-like candidates (this work and Castro et al. 1997) and F and G disk stars (Nissen \& Edvardsson 1992). We found an overabundance for most of our stars compared to their disk counterparts of the same $[\mathrm{Fe} / \mathrm{H}]$. The average $[\mathrm{O} / \mathrm{Fe}]$ of the sample is $+0.17 \mathrm{dex}$ and agrees with the mean value of +0.20 found in Barbuy \& Grenon (1990).

An overabundance of $\alpha$-elements relative to Fe for bulge stars could constrain the formation scenario of the Galatic bulge, since for a fast enrichment by $\mathrm{SNe}$ II an $[\alpha$-element/Fe $]>0.0$ ratio would be expected (Matteucci et al. 1999).

Acknowledgments. L.P. acknowledges the FAPESP PhD fellowship $\mathrm{n}^{o}$ 98/00014-0. We acknowledge FAPESP project $n^{o} 1998 / 10138-8$.

\section{References}

Barbuy, B., \& Grenon, M. 1990, in Bulges of Galaxies, ESO Workshop and Conf. Proc. Vol. 35, eds. B. Jarvis \& D. Terndrup (ESO: Garching), 83

Castro, S., Rich, M. R., Grenon, M., Barbuy, B., \& McCarthy, J. 1997, ApJ, 114,1

Cayrel, R., Perrin, M.-N., Barbuy, B., \& Buser, R. 1991, A\&A, 247, 108

Grenon, M. 1990, in Bulges of Galaxies, ESO Workshop and Conf. Proc. Vol.

35, eds. B. Jarvis \& D. Terndrup (ESO: Garching), 143

Matteucci, F., Romano, D., \& Molaro, P. 1999, A\&A, 341, 458

Nissen, P.E., \& Edvardsson, B. 1992, A\&A, 261, 255 


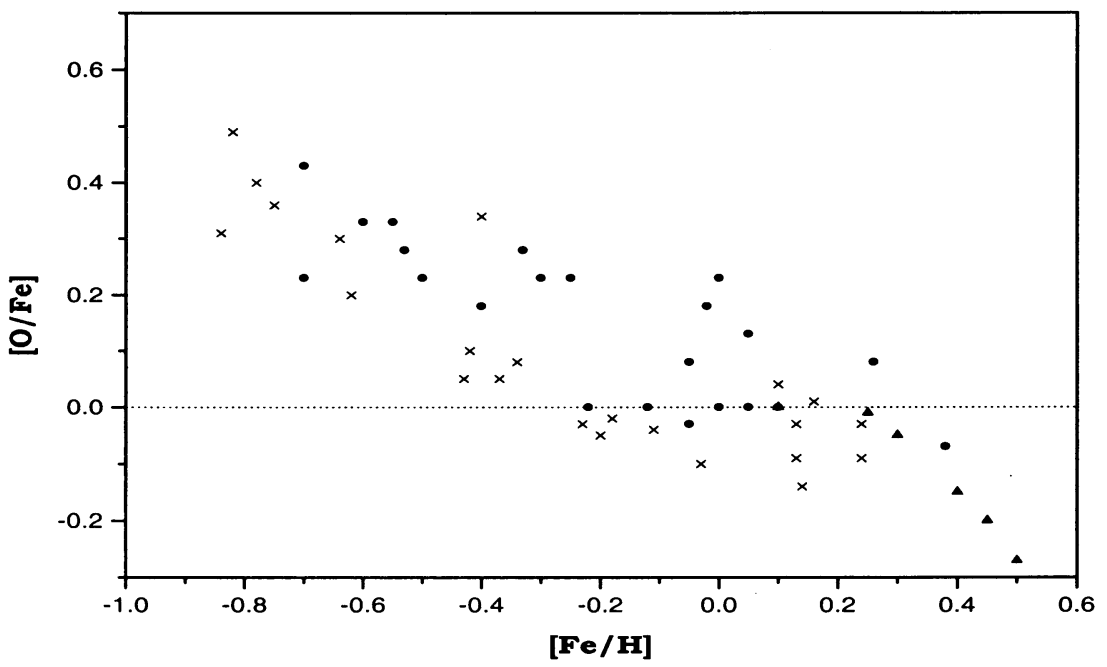

Figure 2. $\quad[\mathrm{O} / \mathrm{Fe}]$ vs. $[\mathrm{Fe} / \mathrm{H}]$ : (filled circles) our data, (filled triangles) Castro et al. (1997), and (crosses) Nissen \& Edvardsson (1992).

Table 2. Oxygen Abundances

\begin{tabular}{crrr}
\hline Star & {$[\mathrm{O} / \mathrm{Fe}]$} & Star & {$[\mathrm{O} / \mathrm{Fe}]$} \\
\hline \hline HD 143016 & +0.23 & HD 214059 & - \\
HD 143102 & 0.00 & CD-4015036 & - \\
HD 148530 & +0.23 & HD 219180 & - \\
HD 149256 & +0.08 & HD 220536 & 0.00 \\
HD 152391 & 0.00 & HD 220993 & +0.23 \\
HDE326583 & +0.23 & HD 224383 & +0.18 \\
HD 175617 & - & HD 4308 & +0.18 \\
HD 178737 & +0.28 & HD 6734 & +0.28 \\
HD 179764 & +0.13 & HD 8638 & +0.23 \\
HD 181234 & -0.07 & HD 9424 & 0.00 \\
HD 184846 & +0.23 & HD 10576 & 0.00 \\
BD-176035 & 0.00 & HD 10785 & - \\
HD 198245 & - & HD 11306 & +0.33 \\
HD 201237 & +0.08 & HD 11397 & +0.13 \\
HD 211276 & - & HD 14282 & - \\
HD 211532 & +0.43 & HD 16623 & - \\
HD 211706 & - & BD-02 602 & - \\
HD 21543 & +0.33 & & \\
\hline
\end{tabular}

